\title{
Fish swimming kinematics in a turbulent wake: To spill or not to spill?
}

\author{
Valentine Muhawenimana $^{1 *}$, Catherine A. M. E. Wilson ${ }^{1}$, Jo Cable $^{2}$ \\ ${ }^{1}$ School of Engineering, Cardiff University, Cardiff, CF24 3AA, UK \\ ${ }^{2}$ School of Biosciences, Cardiff University, Cardiff, CF10 3AX, UK \\ *Corresponding author: MuhawenimanaV@cardiff.ac.uk
}

\begin{abstract}
Due to the complex nature of fish-environment interactions, the effects of altered flow conditions on fish habitats and behaviour, particularly near obstructions, are not yet fully understood. Many hydro-engineering structures generate three-dimensional turbulent structures that differ in properties from naturally occurring ones, and are challenging to fish movement. Here, swimming stability and habitat usage of Nile tilapia (Oreochromis niloticus) were examined in the turbulent wake of a horizontally oriented cylinder (50 mm diameter, D) for a series of increasing cylinder Reynolds numbers $\left(\mathrm{Re}_{\mathrm{d}}\right)$. Velocity statistics showed that the near wake downstream of the cylinder within a $2 \mathrm{D}$ distance had higher magnitudes of longitudinal, vertical and lateral velocity components as well as Reynolds stresses, turbulence intensity and turbulent kinetic energy than the remaining wake flow field. Flow accelerating over the cylinder top and underside generated two shear horizontal layers, where two coherent structures were formed due to the shear layer breakdown off the cylinder's walls and alternating von Karman-type vortex shedding occurred in the wake of the cylinder. The eddy symmetry and vorticity off the cylinder's edges was dependent on $\mathrm{Re}_{\mathrm{d}}$. The recorded total number of spills, defined as loss of balance, was inversely proportional to fish length and weight, and depended on proximity to the cylinder and the flume bed. Furthermore, the frequency of spills was closely linked to the orientation of vortices and magnitude of Reynolds stresses, suggesting that these parameters may govern the swimming stability of fishes. This information may be used to inform the design of fish-friendly obstacles including hydraulic structures and hydro turbines in riverine and estuarine systems.
\end{abstract}

Key words: turbulent wake, fish swimming kinematics, turbulence and fish interactions.

\section{Introduction}

The hydrodynamics associated with engineering obstructions are complex and their impact on fish habitat and fish behaviour is not yet entirely understood. Fish generally prefer predictable flow properties, both spatially and temporally, depending on the size and species of the fish as well as the range of velocities and Reynolds numbers [1-10]. Flow characteristics of velocity and turbulence can also impact fish swimming kinematics and behaviour [5, 8-16], but these effects are unpredictable. Turbulence may have positive effects by generating Karman gait structures that are beneficial to upstream fish movement $[3,7]$. Negligible effects have also been reported where the turbulence has little to no effect on the 
habitat and fish behaviour [4]. Conversely, negative effects of turbulence are more widely reported, with impacts on physiology, energy costs and swimming kinematics [7]. The length scale, direction [5] and diameter of eddies generated from horizontal cylinders in particular [9], have been predicted to govern swimming stability.

The current study assessed the velocity and turbulence metrics that govern fish swimming kinematics and habitat preferences in the wake of a horizontally orientated cylinder. Horizontal cylinders have been shown to have a greater impact on swimming stability and swimming performance than vertical cylinders [9]. This study focuses on a more extensive investigation of the flow field in the confined wake of a cylinder using model fish, which varied in length, over a wider range of cylinder Reynolds numbers than previous studies.

\section{Materials and Methods}

\subsection{Test area}

Experiments were conducted in an open channel recirculating flume (10 m length, $0.3 \mathrm{~m}$ wide and $0.3 \mathrm{~m}$ height) in the Hydraulics laboratory of Cardiff School of Engineering. Flow was powered by a 301/s capacity pump, which draws water from a reservoir at the downstream end of the flume. The horizontal cylinder of diameter (D) $50 \mathrm{~mm}$ was positioned $6 \mathrm{~m}$ from the upstream end of the flume, marking the start of the $0.3 \mathrm{~m}$ long test section, bounded upstream- and downstream by honeycomb flow straighteners. The cylinder was placed transversally across the width of the flume in order to generate turbulent vortices that rotate about a horizontal axis in its wake (see Figure 1).

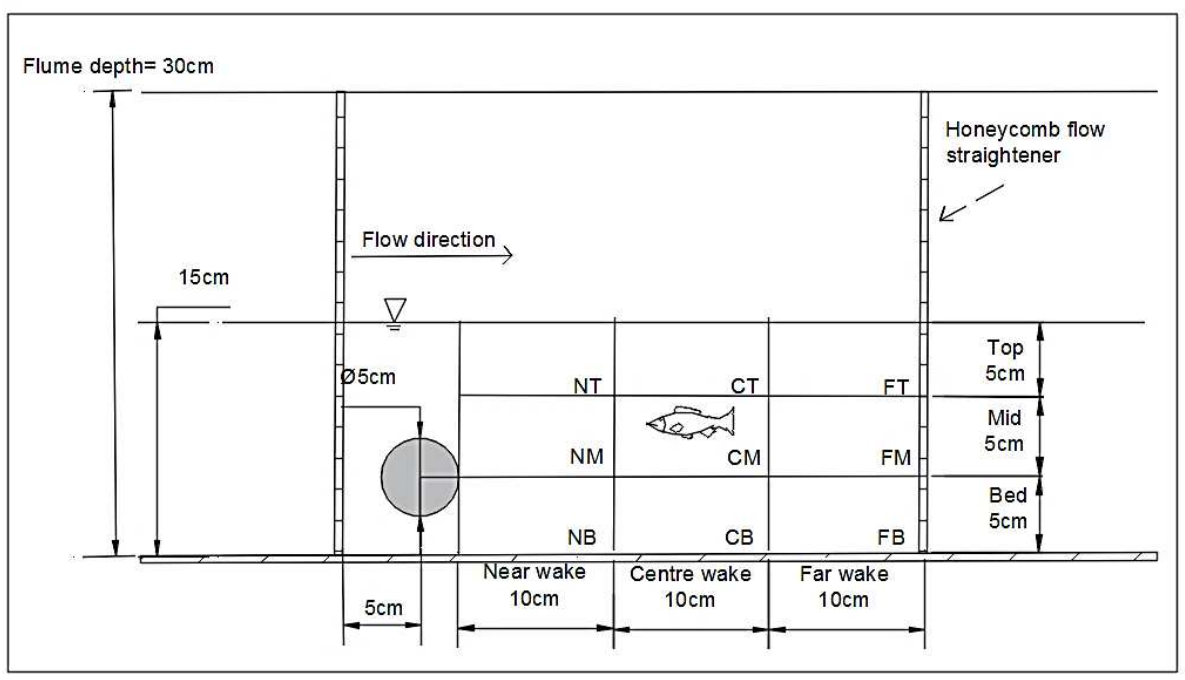

Fig. 1. Experiment test section for fish observation, with dimensions of flow zone volumes that are subdivided by distance from the cylinder (x-direction) and vertical distance away from the bed (z-direction). Zones are characterised: NB is the Near wake Bed zone; NM the Near wake, Mid-water column zone, and NT is the Near wake, Top section in the water column. Likewise, CB, CM, CT, FB, FM, and FT are the bed, mid, and top sections in the Centre and Far wakes respectively.

Flow depth $(\mathrm{H})$ was maintained at $0.15 \mathrm{~m}$ using a tailgate weir located at the downstream end of the flume. The flow rate varied from 3 to $271 / \mathrm{s}$ and increased in increments of $3 \mathrm{Ls}^{-1}$ every $10 \mathrm{~min}$, resulting in cross-sectional averaged velocities ranging from $0.06 \mathrm{~ms}^{-1}$ to 0.60 
$\mathrm{ms}^{-1}$ and cylinder diameter Reynolds numbers $\left(\mathrm{Re}_{\mathrm{d}}\right)$ in the $2.8^{*} 10^{3}$ to $25.8^{*} 10^{3}$ range as shown in Table 1.

Table 1. Velocity step test with time increments (each $10 \mathrm{~min}$ ) and corresponding ranges of cross-sectional averaged velocity and cylinder Reynolds numbers.

\begin{tabular}{|c|c|c|c|}
\hline $\begin{array}{c}\text { Time } \\
\text { [minutes] }\end{array}$ & $\begin{array}{c}\text { Flow rate } \\
\mathrm{Q}\left[\mathrm{Ls}^{-1}\right]\end{array}$ & $\begin{array}{c}\text { Cross-sectional mean } \\
\text { velocity } \mathrm{U}\left[\mathrm{ms}^{-1}\right]\end{array}$ & $\begin{array}{c}\text { Cylinder Reynolds } \\
\text { Number Re }\end{array}$ \\
\hline $0-10$ & 3 & 0.07 & 2,870 \\
\hline $10-20$ & 6 & 0.13 & 5,750 \\
\hline $20-30$ & 9 & 0.20 & 8,620 \\
\hline $30-40$ & 12 & 0.27 & 11,500 \\
\hline $40-50$ & 15 & 0.33 & 14,400 \\
\hline $50-60$ & 18 & 0.40 & 17,200 \\
\hline $60-70$ & 21 & 0.47 & 20,100 \\
\hline $70-80$ & 24 & 0.53 & 23,000 \\
\hline $80-90$ & 27 & 0.60 & 25,900 \\
\hline
\end{tabular}

\subsection{Fish behaviour methodology}

Twenty-eight Tilapia (Oreochromis niloticus, Silver strain), Fork length (117.8 $\pm 21.1 \mathrm{~mm})$, Standard length $(95.2 \pm 17.7 \mathrm{~mm})$ and weight $(31.6 \pm 17.5 \mathrm{~g})$ were maintained at $25^{\circ} \mathrm{C}$ in aquaria in the School of Biosciences, Cardiff University. Individual fish were acclimatized in the flume at $25^{\circ} \mathrm{C}$ for $30 \mathrm{~min}$ at the lowest flow rate of $1.51 / \mathrm{s}$ before the start of the experiment. The acclimation section was located immediately downstream of the observation section, delimited by honeycomb flow straighteners. At the start of the experiment, fish were allowed to swim to the observation section by moving the separation flow straightener. The flow rate was then gradually increased to the first flow rate of the experiment and maintained for an observation time of $10 \mathrm{~min}$, after which the flow rate was increased from $31 \mathrm{~s}^{-1}$ to $271 \mathrm{~s}^{-}$ ${ }^{1}$ in flow rate increments of $3 \mathrm{Ls}^{-1}$. Each flow rate step was maintained for $10 \mathrm{~min}$ of fish swimming kinematics observations, which were recorded using two Logitech C920 1080p HD web cameras that were set up on the glass sides of the flume with the entire observation section in frame. The videos were later reviewed using JWatcher V1.0 software to quantify the fish swimming behaviour and habitat usage by logging the time and place of spill occurrence as well as the amount of time fish spent in each zone of the test window. Spills, defined following [9] are events of balance loss, where the head of the fish rotates more than $45^{0}$, the fish drifts downstream by more than 0.5 its total body length; followed by the fish recovering from the spill by reorienting its body to the flow. The frequency of spills $\left(\mathrm{min}^{-1}\right)$ was defined as the ratio of the number of spills per zone over the amount of time spent in that flow volume zone.

\subsection{Velocity measurements and analyses}

Spatial and temporal resolution ADV measurements were collected in the wake of the cylinder using an Acoustic Doppler Velocimeter, Nortek Vectrino (V.1.31+) at a sampling rate of $200 \mathrm{~Hz}$. The velocities were measured in three dimensions and reported in xyz coordinates, where $\mathrm{x}$ is in the longitudinal direction of the flow, $\mathrm{y}$ is in the transverse direction and $\mathrm{z}$ is in the vertical direction. Fifteen points were measured in the $\mathrm{z}$ direction with the first point $10 \mathrm{~mm}$ away from the flume bed, and points thereafter equispaced by $5 \mathrm{~mm}$. Each point was measured for a sampling time of $300 \mathrm{~s}$ each. Fifteen vertical profiles were measured in 
the (x) longitudinal direction in increments of $20 \mathrm{~mm}$. Using Velocity Signal Analyser V1.5.62 MAJVSA, the velocity data was filtered with thresholds of 15 for sound to noise ratio (SNR) and 70\% for Correlation. Additional despiking used 12-point average spike replacement and the Modified Phase-Space Thresholding method [17-18].

\section{Results}

\subsection{Turbulent Wake Dynamics}

In the cylinder wake a low velocity recirculation zone is present and two horizontal shear layers were generated as the flow accelerates over the cylinder topside and underside of the cylinder. Two coherent structures were formed due to the shear layer breakdown off the cylinder's wall and alternating von Karman vortex shedding occurred in the wake of the cylinder. The vortices were symmetric at the lowest $\operatorname{Re}_{\mathrm{d}}\left(\operatorname{Re}_{\mathrm{d}}=2,870\right)$ and became increasingly asymmetric with increasing $\mathrm{Re}_{\mathrm{d}}$ due to the presence of the bed wall. The turbulent vortices continually dissipated with increasing downstream distance from the cylinder. Across all test section zones, spatially-averaged turbulent kinetic energy and shedding frequency increased with increasing $\mathrm{Re}_{\mathrm{d}}$, while turbulence intensity decreased with increasing $\mathrm{Re}_{\mathrm{d}}$. In addition, the near wake had higher magnitudes of longitudinal, vertical and lateral velocity components as well as Reynolds stresses and turbulent kinetic energy compared to the centre and far wakes for all $\mathrm{Re}_{\mathrm{d}}$.

(A)

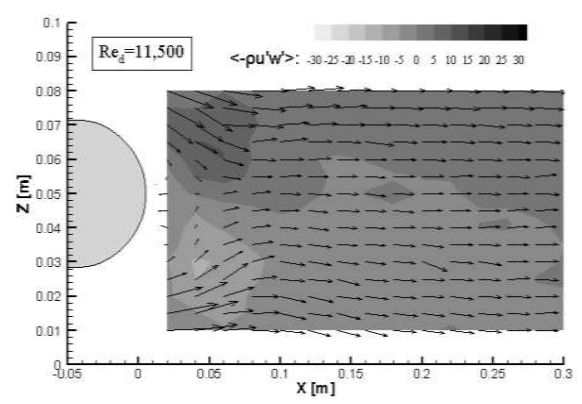

(B)

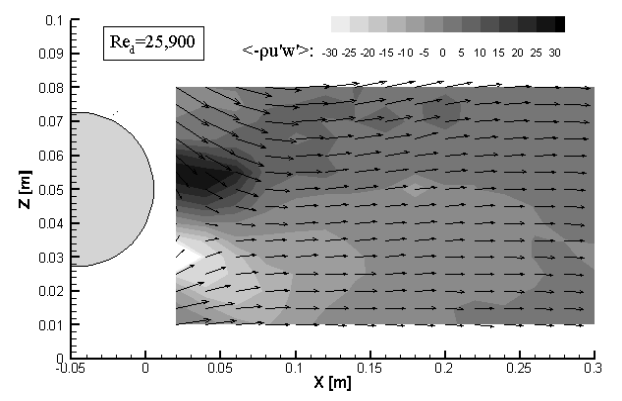

Fig. 2. Contours of the vertical Reynolds stress $<-\rho u^{\prime} w^{\prime}>\left(\mathrm{Nm}^{-2}\right)$ superimposed with the timeaveraged velocity vectors $(\mathrm{u}, \mathrm{w})$ for cylinder Reynolds numbers $\operatorname{Re}_{\mathrm{d}}(\mathrm{A}) 11,500$ and $(\mathrm{B})$ 25,900 . The cylinder wake is characterized by a recirculation zone immediately in the lee of the cylinder, and under- and upper-flow accelerations, which generate the two horizontal shear layers.

\subsection{Fish behaviour}

Habitat usage characterized in terms of the time spent in each zone did not vary significantly with increasing Reynolds number. Fish spent the most time station holding (55.5\%) in the FB flow volume zone followed by CB volume (17\%) which were characterized by the lowest turbulence intensity and turbulent kinetic energy of the test section and the least amount of time was spent in the NM $(1.7 \%)$ and NT $(1.4 \%)$ zones. Overall, the habitat preference in terms of amount of time spent in a particular zone increased with increasing distance from the cylinder and decreased with increasing distance from the flume bed. In total, 529 spills were recorded from all tests, and the number of spills per fish was inversely proportional to 
the fish length and weight, with the longer fish experiencing less spills. Fewer spills occurred to fish station holding increasingly further from the cylinder and the flume bed. Furthermore, the number of spills increased with increasing $\mathrm{Re}_{\mathrm{d}}$, reaching a maximum at a $\mathrm{Re}$ of 11,500 after which the number of spills decreased for the remaining $R_{d}$.

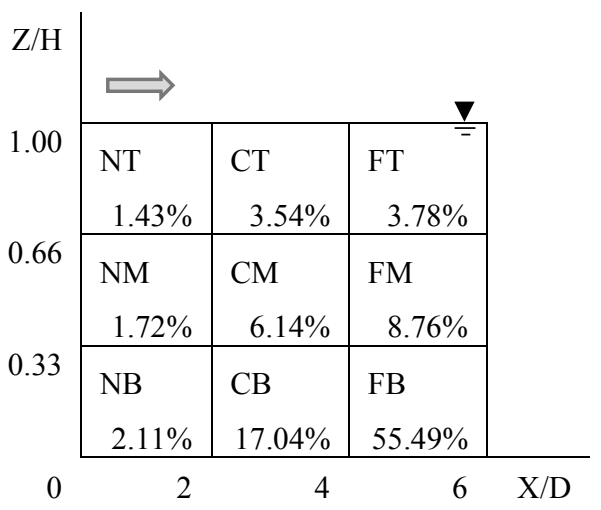

(A)

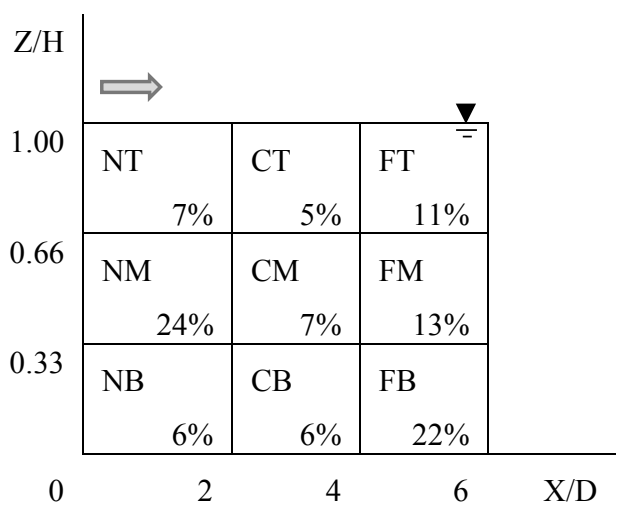

(B)

Fig 3. Percentage of (A) time spent and (B) spill distribution in each zone. Zones are outlined in Figure 1.

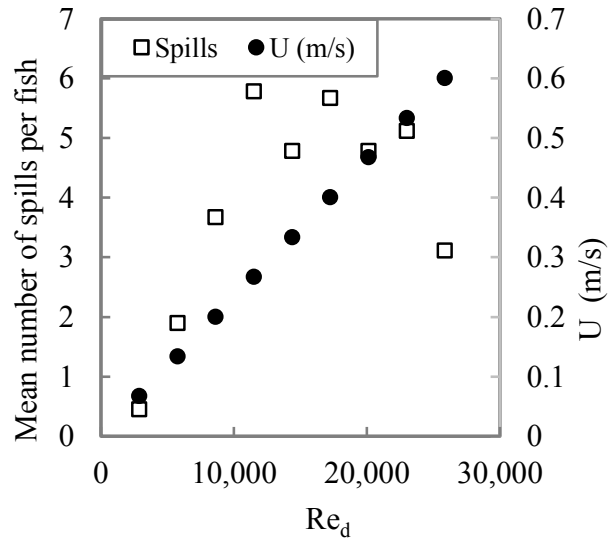

(A)

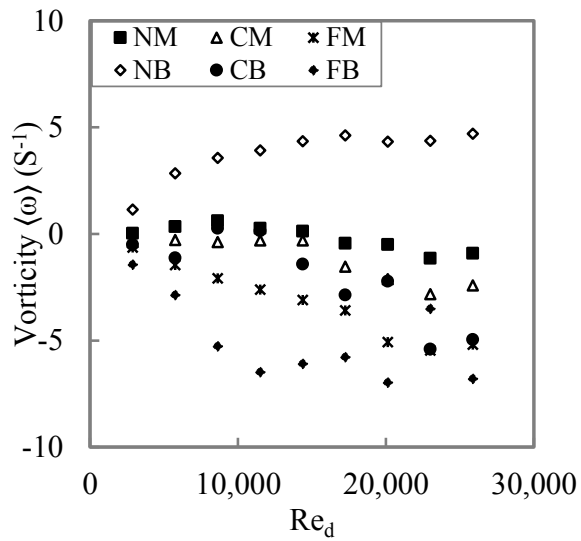

(B)

Fig 4. (A) Mean number of spills gradually increased with increasing $\mathrm{Re}_{\mathrm{d}}$ and reached a maximum value at $\mathrm{Re}_{\mathrm{d}}=11,500$ then decreased for the remaining $\mathrm{Re}_{\mathrm{d}}$ conditions. Crosssectionally averaged velocity $U(\mathrm{~m} / \mathrm{s})$ increased linearly with increasing $\mathrm{Re}_{\mathrm{d}}$. (B) Z-plane vorticity with increasing $\mathrm{Re}_{\mathrm{d}}$ where $\mathrm{NB}$ zone is dominated by positive clockwise vortices of high vorticity for all $\mathrm{Re}_{\mathrm{d}}$, whereas vortices in $\mathrm{NM}$ changed from clockwise (+ve vorticity) to counter-clockwise (-ve) vortices after $\mathrm{Re}_{\mathrm{d}}=14,400$. The remaining zones all have negative clockwise vortices with FB being the highest in magnitude for all examined $\mathrm{Re}_{\mathrm{d}}$.

The highest number of spills $23.6 \%$, occurred in the Near wake, mid-water column zone (NM) followed by the Far wake zone (FB) where $21.9 \%$ of all spills occurred. Other zones NB, NT, CB, CM, CT, FM, and FT accounted for $6 \%, 6.6 \%, 5.9 \%, 7.4 \%, 4.9 \%, 12.7 \%$ and $11 \%$ of spills respectively (Figure 3 ). The number of spills was inversely proportional 
to fish length and weight, where the smallest fish experienced significantly more spills than longer ones. In the $\mathrm{X}$ direction, the number of spills was highest in the far wake volume, followed by the near wake and least in the Centre wake zones. The number of spills was also highest in the mid-water column zone in the $\mathrm{Z}$ direction, followed by the zones nearest to the bed and lowest in the top layer of the flow depth. For all fish, the frequency of spills was highest in the near wake, decreased with increasing longitudinal distance from the cylinder and increased with increasing vertical distance from the bed. Downstream of the zones in the near wake, with the exception of the highest $\mathrm{Re}_{\mathrm{d}}$ flow condition, the frequency of spills remained relatively constant with increasing $\operatorname{Re}_{\mathrm{d}}$ (see Figure 5).

\section{Discussion}

Although fish spent the least time in the NM zone, near wake and mid-water column, the highest number of spills occurred in this zone, making it the highest in frequency of spills (spills per min). In the zone where fish preferred to station hold (FB, far wake within 1D of the bed), there were a proportional high number of spills, making the ratio of spills number/time (frequency) to be one of the lowest. This suggests that the fish preferred the zones where spills were least likely to occur as evidenced by the low frequency of spills. The FB, FM and FC zones, where the cylinder generated vortices had dissipated, were characterized by lower velocities, turbulent kinetic energy, and low Reynolds shear stresses, but had higher turbulence intensities. Habitat usage did not significantly vary with increasing $\mathrm{Re}_{\mathrm{d}}$, since the relative velocity and turbulence metrics were similarly distributed in the cylinder wake, making the preferred volume zones consistently favorable for the fish. The increase in the number of spills up to an intermediate $\mathrm{Re}_{\mathrm{d}}$ of 11,500 may point to a change in cylinder wake dynamics and this requires further investigation.

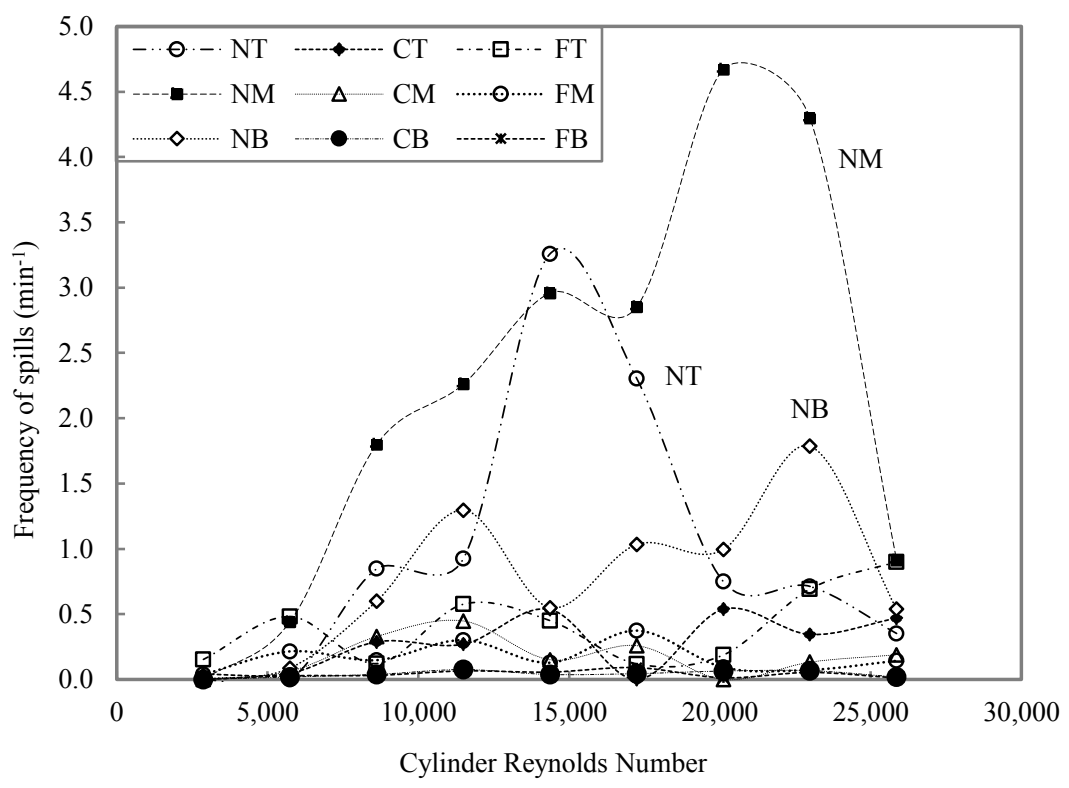

Fig. 5. Variation of frequency of spills $\left(\mathrm{min}^{-1}\right)$ per $\mathrm{Re}_{\mathrm{d}}$ and flow volume zones shows that the near wake zones had the highest ratio of spill occurrence over amount of time the fish spent in the zone, and this frequency changed with increasing $\mathrm{Re}_{\mathrm{d}}$. The Centre and Far wake zones where fish preferred to station hold show little to no variation in frequency of spills over the range of $\mathrm{Re}_{\mathrm{d}}$. 
The zones with the highest frequency of spills, NM and NB not only have the highest magnitudes of vertical and horizontal Reynolds shear stresses (Fig. 2) but also positive or negligible vorticity (Fig. 6) which most likely resulted in overturning moments that destabilized the fish [5]. However, the Centre and Far wake zones with predominantly negative vorticity resulted in lower spill frequency despite having similar vorticity, but with lower Reynolds stresses. Longitudinal turbulent length scale, calculated using the autocorrelation function [20], reveal that the ratio of turbulence length scale to cylinder diameter $\left(\mathrm{L}_{\mathrm{u}} / \mathrm{D}\right)$ were similar in range across zones with the highest spill frequency NM, and NB as well as zones with lower frequency of spills (Fig. 7).

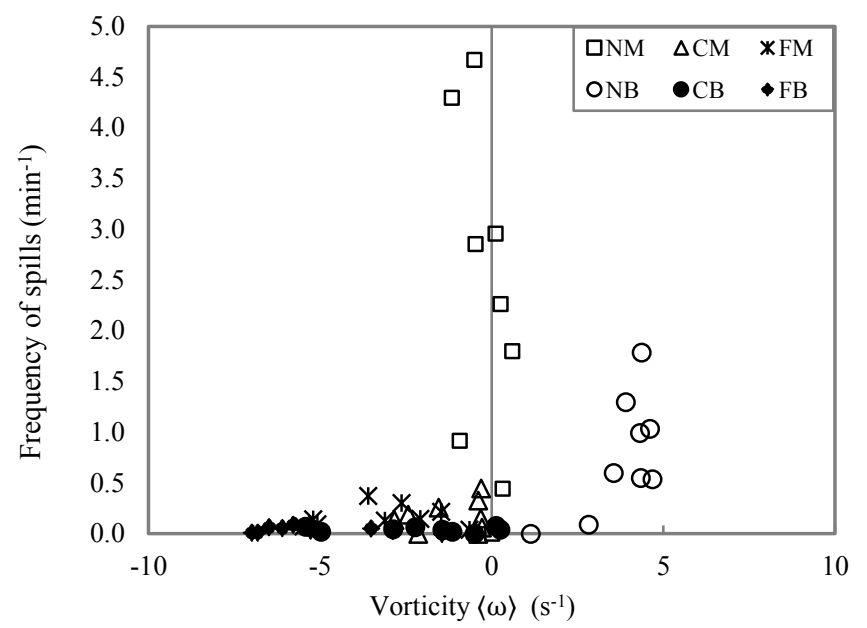

Fig. 6. Frequency of spills relative to the spatially-averaged vorticity per zone shows that higher frequency of spills correspond to zones with positive vorticity and negligible vorticity while negative vorticity results in significanlty less spills per min.

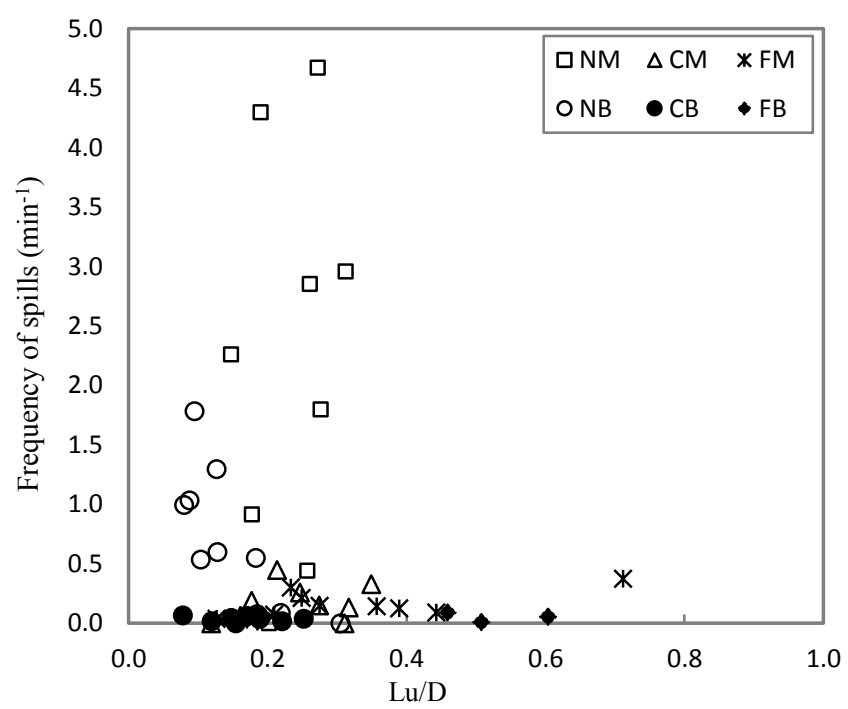

Fig. 7. Frequency of spills relative to the mean turbulent length scale per zone shows that the majority of $\mathrm{L}_{\mathrm{u}} / \mathrm{D}$ values result in a varied range of frequencies of spills. 


\section{Conclusion}

The zones immediately behind the cylinder, in which fish experienced the highest frequency of spills, showed the highest magnitudes of vertical and horizontal Reynolds shear stresses and positive vorticity, which most likely resulted in overturning moments that destabilized the fish. The Reynolds shear stresses and the direction of rotation of the vortices, rather than eddy magnitude and length scale, may govern the occurrence of spills, as counter-clockwise vortices and higher Reynolds stress resulted in a higher frequency of spills. Further investigation into linkages between the different velocity and turbulence metrics and fish swimming kinematics will define the key factors that regulate these interactions. This information may be used to inform the design of fish-friendly obstacles including hydraulic control structures and hydro turbines in riverine and estuarine systems.

\section{References}

1. D.S. Pavlov, A. I. Lupandin, M.A. Skorobogatov. J. Ichthyol 40, 232-261 (2000)

2. P.W. Webb, A.J. Cotel. Fish Physiol Biochem 37, 297-305 (2011)

3. E.C. Enders, T. Buffin-Bélanger, D. Boisclair, A.G. Roy. Can. J. Fish. Aquat. Sc. 66, 242-253 (2003)

4. V.I. Nikora, J. Aberle, B.J.F. Biggs, I.G. Jowett, J.R.E. Sykes. J. Fish Biol 63, 1365$1382(2003)$

5. A.I. Lupandin. Biol. Bull. 32, 558-565 (2005)

6. A.J. Cotel, P.W. Webb, H.M. Tritico. Trans. Am. Fish. Soc. 135, 610-619 (2006)

7. J.C. Liao. Philos. Trans. Roy. Soc. B 362, 1973-1993 (2007)

8. D.S. Pavlov, A.I. Lupandin, M.A. Skorobogatov. J. Ichthyol 40, 232-261 (2000)

9. H.M. Tritico, A.J. Cotel. J. Exp. Biol. 213, 2284-2293 (2010)

10. F.A. Hockley, C.A.M.E. Wilson, A. Brew, J. Cable. J. R. Soc. Interface 11: 20130814. (2014)

11. A.T. Silva, J.M. Santos, M.T. Ferreira, A.N. Pinheiro, C. Katapodis. River Res. Appl. 27, 360-373 (2011)

12. J.C. Liao, D.N. Beal, G. V. Lauder, M.S. Triantafyllou. Science 302, 1566 (2003)

13. J.C. Liao, D.N. Beal, G. V. Lauder, M.S. Triantafyllou. J. Exp. Biol. 206, 1059-1073.

14. P. Webb. J. Exp. Biol., 201, 2403-2412 (1998)

15. P.W. Webb. Integ. And Comp. Biol. 42, 94-101 (2002)

16. D.L. Smith, E.L. Brannon, B. Shafii, M. Odeh. Trans. Am. Fish. Soc. 135, 431-441 (2006)

17. D.G. Goring, V.I. Nikora. J. Hydraul. Eng. 128(1), 117-126 (2002)

18. T.L. Wahl. Building Partnership. 1-10. (2000)

19. J.F. Douglas, J.M. Gasiorek, J.A. Swaffield, L.B. Jack. Fluid Mechanics $6^{\text {th }}$ ed. pp.219. (2011)

20. S.B. Pope. Turbulent Flows. pp.68-71 (2000) 